\title{
A Female Urethral Melanoma Treated with a Bladder Sparing Approach
}

\begin{abstract}
Keywords: Bladder - sparing surgery; Urethrectomy; Mucosal melanoma; Urethral mass; Metastatic melanoma

Abstract

Malignant melanoma rarely involves the genitourinary organs, however urethral involvement accounts for the majority of documented cases. In comparison to cutaneous melanoma, urethral melanoma is associated with a poor prognosis owing to the advanced stage at presentation. Treatment options are largely dictated by the extent of disease, however diagnostic and treatment recommendations are not clearly defined. Surgical options can range from distal urethrectomy, to radical cystectomy for those lesions involving the bladder neck. Although local control can be achieved with radical extirpative surgery, the effect on the patient's urinary control and the patient's quality of life are detrimental. In the following case, we present a 68-year-old Asian American woman with advanced urethral melanoma who was treated with distal urethrectomy, radical hysterectomy, and adjuvant chemotherapy. Using this multimoda treatment the patient was able to enjoy a satisfactory quality of life with no evidence of disease recurrence at two years after treatment.
\end{abstract}

\section{Introduction}

Genitourinary malignant melanoma is a rare form melanoma that includes mucosal melanomas of the urethra. Urethral melanoma accounts for the majority of cases of genitourinary melanoma and is associated with a poor prognosis in comparison to cutaneous melanomas [1,2]. Being a rare disease, diagnostic and treatment recommendations are not clearly defined in the literature and as a result treatment decisions may be difficult in regards to providing adequate oncologic control while preserving genitourinary function. Herein we present a case of malignant urethral melanoma with a synchronous cervical lesion in a patient where local disease control was achieved while preserving urinary function.

\section{Case Presentation}

The patient is a 68-year-old Asian-American woman with a chief complaint of pelvic floor pressure and pain, dysuria, and strangury. Several weeks prior to referral she noticed blood on her undergarments and began using 1-2 sanitary pads daily. She did not have any additional complaints, and her past urologic, gynecological, and social history was noncontributory. Her past medical history was significant for Non-Hodgkins lymphoma involving the nasopharynx, and had been in remission for fifteen years after chemotherapy with cyclophosphamide, hydroxydaunorubicin, vincristine, and prednisolone (CHOP). Physical examination was remarkable for anexophytic, pedunculated soft tissue mass emanating from the urethral meatus (Figure 1). The lesion was darkly pigmented, and did not involve the bladder neck. On pelvic examination there was a flat pigmented lesion found at the cervical os, and no remaining mucosal lesions in the vagina. There was no palpable lymphadenopathy and

\section{Journal of}

Urology \& Nephrology

\section{Ricardo Palmerola* and Michael Schwartz}

The Arthur Smith Institute for Urology, Hofstra Northwell School of Medicine, New Hyde Park, New York, USA

Address for Correspondence

Ricardo Palmerola, The Arthur Smith Institute for Urology, Hofstra Northwell School of Medicine, 450 Lakeville Rd, Suite M41, New Hyde Park, New York 11042, USA, Tel: 516-734-8500; Fax: 516734-8535; Email: ricardopalmerola@gmail.com

Submission: 12 October, 2016

Accepted: 14 November, 2016

Published: 21 November, 2016

Copyright: ๑ 2016 Palmerola R, et al. This is an open access article distributed under the Creative Commons Attribution License, which permits unrestricted use, distribution, and reproduction in any medium, provided the original work is properly cited.

the remainder of the exam was unremarkable. Laboratory tests were unremarkable including complete metabolic panel, complete blood count, urinalysis, urine cytology and urine culture. Cystourethroscopy revealed no more proximal urethral involvement of the pigmented mass and there were no urothelial lesions in the lower urinary tract. A biopsy of the urethral mass was performed and the patient was referred to gynecology for further evaluation of the cervical lesion. The urethral biopsy revealed a specimen with histologic findings consistent with a malignant melanoma and subsequent immunohistochemical staining including HMB-45 and S-100 were positive [3-5]. Evaluation by dermatology and ophthalmology revealed no evidence of ocular or cutaneous primary lesions. Computed tomography of the chest, abdomen, and pelvis was unremarkable. MRI (Magnetic Resonance Imaging) of the pelvis was utilized to assess the soft tissue extension of the primary tumor, and rule out bladder neck involvement. The patient's MRI demonstrated an enhancing mass confined to the

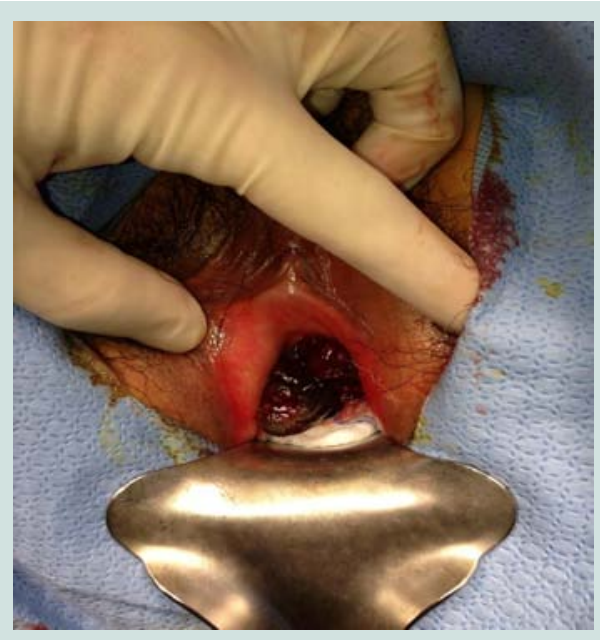

Figure 1: Urethral Melanoma. 


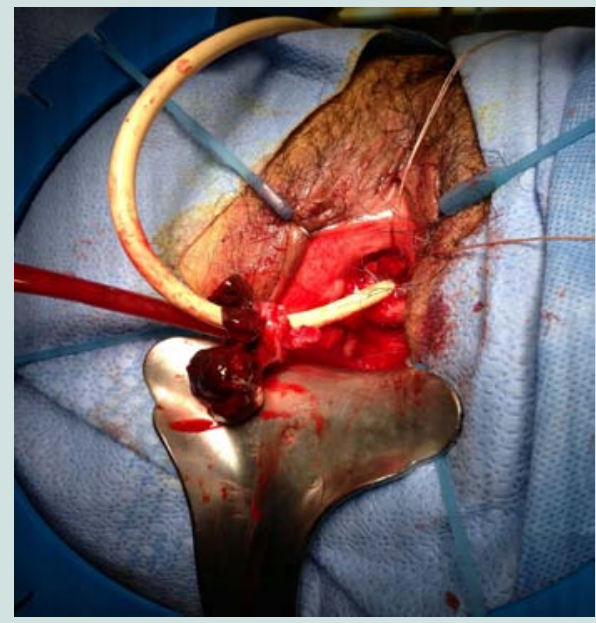

Figure 2: Distal urethrectomy specimen.

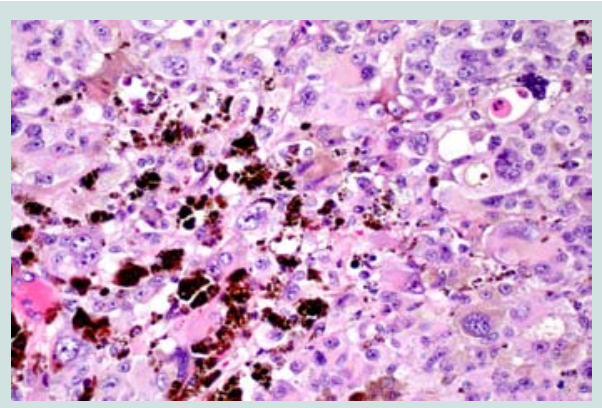

Figure 3: Malignant melanoma. Melanocytic cells with high mitotic rate are demonstrated.

periurethral fascia not involving the bladder neck. A PET (Positron Emission Tomography) scan was performed prior to intervention to identify the presence of metastatic disease not previously identified on cross sectional imaging. The patient's PET scan identified hyperactivity confined to the cervix and the distal urethra.

The patient underwent an uneventful cervical biopsy under anesthesia and distal urethrectomy. Distal urethrectomy was performed with an approximately $1 \mathrm{~cm}$ urethral margin with no evidence of disease on frozen section of the anterior vagina (Figure 2). Final pathology demonstrated malignant melanoma in situ (Tis) with negative proximal urethral margins (Figure 3). Additionally, cervical biopsies also demonstrated malignant melanoma and she underwent a subsequent radical hysterectomy, bilateral salpingooophorectomy with proximal vaginectomy. The final specimen demonstrated malignant melanoma confined to the cervix with less than $1 \mathrm{~cm}$ invasion,no ulceration, and negative vaginal margins (T1). The postoperative course was uneventful and she received six cycles of adjuvant cisplatin and temozolomide. Postoperatively, she was followed with routine pelvic examinations, cystoscopy, and PET imaging. Postoperatively, she was followed with routine pelvic examinations, cystoscopy (every 6-9 months), and PET imaging (annually). At 2 years follow-up she had normal urinary function without incontinence. Furthermore, she had no evidence of disease on physical exam, cystoscopy or PET/CT scan.

\section{Discussion}

Urethral malignant melanoma was first reported over a century ago with approximately 150 cases reported to date $[1,3]$. The etiology of urethral and mucosal melanomas remains inconclusive with no clear environmental or genetic risk factors elucidated. Urethral melanoma is more common in women, and the mean age is approximately 64 years of age [1]. Presenting symptoms can include obstructive or irritative lower urinary tract symptoms, hematuria, perineal pain, and vaginal/urethral bleeding [3]. On physical exam the lesion may grow in a nodular, polypous, or papillary pattern and the color may range from amelanotic to dark pigmented lesions [2,3]. Urethral melanoma is classically associated with a poor prognosis, owing to its early dissemination via the urethra's rich vascular and lymphatic supply. Additionally, delayed patient presentation and misdiagnosis for similar appearing urethral lesions (urethral prolapse, caruncle, and polyps) contribute to advanced disease upon diagnosis [2]. Thus the initial step in the work up of our patient included establishing a tissue diagnosis and proceeding with a thorough evaluation for metastatic disease.

The extent of local invasion and metastatic disease is often underestimated with clinical staging. For example, DiMarco found over $70 \%$ of the patients in their series were upstaged from clinical T2 urethral melanoma cancer to pT3 in their series et al. [2]. As a result, wide local excision, often involving anterior exenteration to provide local control of disease is recommended. Our approach relied upon diagnostic imaging modalities to assess the patient's eligibility for bladder preservation. In addition to utilizing CT for metastatic evaluation, we used MRI to assess local tumor spread to periurethral tissue and the bladder neck. Similar to the workup of cutaneous melanoma, obtaining a PET/CT scan was useful in determining disease localization in our patient to the distal urethra and uterine cervix and also for the purposes of follow up.

The main stay of treatment for distal female urethral lesions without bladder neck involvement is distal urethrectomy [1-3]. In our case the patient desired to remain continent and based on the preoperative workup performed, the lesion was confined to the distal third of the urethra. Likewise, radical hysterectomy is indicated in the management of localized cervical melanoma [6]. The value of inguinal lymph node dissection has not been defined for urethral melanoma, with one series concluding no difference in outcomes [2]. In this case, there was no evidence of clinical or radiographic lymph node metastasis and therefore lymphadenectomy was deferred.

Despite adequate local resection, published reports have demonstrated high recurrence and progression of disease [2]. The routine use of adjuvant chemotherapy for urethral melanoma lacks significant clinical evidence and has been limited to reported cases in the literature $[1,7,8]$. However, various chemotherapy regimens have been adapted from the management of advanced cutaneous melanoma [1]. More recently, cisplatin based regimens have been investigated and shown efficacy in the management of advanced melanoma. Our choice of chemotherapy was based on a recentphase II randomized clinical trial comparing high dose interferon to temozolomide plus cisplatin. The study included 189 chemotherapy naïve non-metastatic 
patients with stage II and III mucosal melanoma that underwent complete surgical resection. Patients were randomized 1:1:1 to three groups including observation, high dose interferon and temozolomide with cisplatin. Approximately $25 \%$ of the patients in the study were diagnosed with a mucosal melanoma of genitourinary origin. After a median follow up of 26.8 months, the overall survival was more than double in the treatment arm using temozolomide/cisplatin versus observation ( 20 v. 48.7 months, $\mathrm{p}<0.01)$. Temozolomide/cisplatin also out performed high dose interferon in terms of recurrence-free survival $(20.8$ v. 9.4 months, $\mathrm{p}<0.001)$ and overall survival $(48.7$ v. 40.4 months, $\mathrm{p}<0.01$ ) [9].

\section{Conclusion}

In conclusion, bladder preservation is possible in the setting of urethral melanoma with and isolated cervical lesion. An extensive preoperative work up to assess local invasion of the primary lesion, detection of distant metastases, and presence of lymphadenopathy is critical. For lesions localized to the distal urethra without bladder neck invasion, distal urethrectomy may be offered while management of female reproductive organs is best managed with radical excision Adjuvant chemotherapy could be offered because of the high risk of recurrence and/or progression; however there is no established regimen. Finally, routine follow-up and use of PET scan should be considered.

\section{References}

1. El-Safadi S, Estel R, Mayser P, Muenstedt K (2014) Primary malignant melanoma of the urethra: a systematic analysis of the current literature. Arch Gynecol Obstet 289: 935-943.

2. DiMarco DS, DiMarco CS, Zincke H, Webb MJ, Keeney GL, et al. (2004) Outcome of surgical treatment for primary malignant melanoma of the female urethra. J Urol 171: 765-767.

3. Cho ST, Song HC, Cho B, Choi WS, Lee WK, et al. (2012) Primary malignant melanoma of the female urethra. Korean J Urol 53: 206-208.

4. Cochran AJ, Wen DR (1985) S-100 protein as a marker for melanocytic and other tumours. Pathology 17: 340-345.

5. Wick MR, Swanson PE, Rocamora A (1988) Recognition of malignant melanoma by monoclonal antibody HMB-45. An immunohistochemical study of 200 paraffin-embedded cutaneous tumors. J Cutan Pathol 15: 201-207.

6. Cantuaria G, Angioli R, Nahmias J, Estape R, Penalver M (1999) Primary malignant melanoma of the uterine cervix: case report and review of the literature. Gynecol Oncol 75: 170-174.

7. Yoshizawa T, Kawata N, Sato K, Hirakata H, Igarashi T, et al. (2007) Primary malignant melanoma of the female urethra. Urology 70: 1222.

8. Alvarez Kindelán J, Merchan Garcia JA, Olmo Cerezo I, Moreno Rodriguez MM, Gonzalez Arlanzon MM (2000) Primary malignant melanoma of the female urethra. Report of a case. Actas Urol Esp 24: 488-490.

9. Lian B, Si L, Cui C, Chi Z, Sheng X, et al. (2013) Phase II randomized trial comparing high-dose IFN- $\alpha 2 b$ with temozolomide plus cisplatin as systemic adjuvant therapy for resected mucosal melanoma. Clin Cancer Res 19: 44884498. 